Pacific Journal of Mathematics

TS 


\title{
COMPACT CONVEX SETS WITH THE EQUAL SUPPORT PROPERTY
}

\author{
JoHN N. MCDONALD
}

\begin{abstract}
Simplexes may be characterized as follows: (C) $X$ is a simplex if and only if each $x \in X$ has a unique $\prec$-maximal representing measure, where $\prec$ denotes the Choquet ordering on the set $M^{+}(X)$ of positive regular Borel measures on $X$. In this paper, we study compact convex sets which satisfy a condition which is similar to that given in (C). Definition: $X$ has the equal support property if, for each $x \in X$, any two $\prec$-maximal representing measures for $x$ have the same support. Some of our theorems are extensions to sets with the equal support property of results which hold for simplexes. Other results given here are analogous of theorems which hold for simplexes. We are especially interested in the relationships between the equal support property and a topology, called the structure topology, which was first defined for the set of extreme points of a simplex, but also makes sense for a wider class of compact convex sets.
\end{abstract}

The background material for $\S \S 1$ through 4 of this paper may be found in [12]. The equal support property was first considered by Feinberg in [8]. The expression "compact convex set" will always refer to a nonempty compact convex subset of a locally convex Hausdorff linear space. Let $X$ be a compact convex set. ex $X$ will denote the set of extreme points of $X . \partial X$ will denote the closure $\overline{e x} X$ of ex $X$. Consider a point $x \in X$ and a closed subset $F$ of $X$. $R_{x}^{F}$ will denote the set of representing measures for $x$ which are supported by $F$, i.e., vanish on $X-F$. (We will not distinguish between measures on $F$ and measures on $X$ which are supported by $F$.) It is known [12, p. 5] that $y \in \overline{\operatorname{cov}} F$ if and only if $R_{y}^{F} \neq \varnothing$. Recall tha $X=\overline{\operatorname{cov}}(e x \quad X)$ (Krein-Milman Theorem, KMT), or, equivalently, $R_{x}^{\partial X} \neq \varnothing$ for each $x \in X$. We will make use of Milman's converse to the Krein-Milman Theorem, henceforth refered to as $M T$, which states that: $X=\overline{\operatorname{cov}} S$ implies $e x X \subseteq \bar{S}$.

1. Extreme sets. In this section, $X$ will denote a compact convex set. A fuction $f: X \rightarrow Z$ where $Z$ is a convex set is called affine if $f(c x+(1-c) y)=c f(x)+(1-c) f(y)$ for every

$$
(x, y, c) \in X \times X \times[0,1] \text {. }
$$

A function $g: X \rightarrow(-\infty, \infty)$ is called concave if 


$$
c g(x)+(1-c) g(y) \leqq g(c x+(1-c) y)
$$

for all $(x, y, c) \in X \times X \times[0,1]$.

Definition 1.1. A set $S \subseteq X$ will be called extreme if

$$
(u, v, b) \in X \times X \times(0,1)
$$

and $b u+(1-b) v \in S$ imply $u, v \in S$. A convex extreme set will be called a face.

Note the union of any collection of closed faces is extreme. In particular, if $S \subseteq e x X$, then $S$ is extreme.

LEMma 1.2. A closed subset $E$ of $X$ is extreme if and only if $x \in E$ implies supp $\rho \subseteq E$ for each $\rho \in R_{x}^{X}$ (supp $\rho$ denotes the closed support of the measure $\rho$ ).

The proof is a slight modification of the proof of [12, prop. 1.4]. See also, [1, p. 100].

Let $\mathscr{F}(X)$ denote the collection of closed faces of $X$. Note that, if $\mathscr{C} \subseteq \mathscr{F}(X)$, then $\cap\{C \mid C \in \mathscr{C}\} \in \mathscr{F}(X)$. Thus, $\mathscr{F}(X)$ is a complete lattice in the containment ordering. Let $F^{\prime \prime}, F^{\prime \prime} \in \mathscr{F}(X)$. We will denote $\cap\left\{F \mid F \in \mathscr{F}(X)\right.$ and $\left.F \supseteqq F^{\prime} \cup F^{\prime \prime}\right\}$ by $F^{\prime} \vee F^{\prime \prime}$. The following gives some elementary but useful information about members of $\mathscr{F}(X)$ :

Proposition 1.3. Let $F, F^{\prime \prime} \in \mathscr{F}(X)$. Then: (a) ex $F=e x X \cap F$. (b) $e x\left(\operatorname{cov}\left(F \cup F^{\prime}\right)\right)=e x F \cup e x F^{\prime}=e x X \cap \operatorname{cov}\left(F \cup F^{\prime}\right)$.

Proof. (a) is clear. By MT, ex $\left(\operatorname{cov}\left(F \cup F^{\prime}\right)\right) \subseteq F \cup F^{\prime \prime}$. now follows from (a).

It is natural to ask, whether $F, F^{\prime} \in \mathscr{F}(X)$ imply cov $\left(F \cup F^{\prime}\right) \in \mathscr{F}(X)$ ? A more general question is: If $E$ is closed and extreme, is $\overline{\operatorname{cov}} E$ extreme? It follows from a result of Effros that, if $X$ is a simplex and $E \leqq e x X$, then the answer to the last question is yes (see [7, Th. 3.3]). The following theorem shows that the answer to the above question is affirmative if $X$ has the equal support property, furthermore, the proof given here may be used to obtain Th. 3.3 of [7]:

TheOREM 1.4. Suppose that $X$ has the equal support property. Let $E$ be a closed extreme set of $X$. Then $\overline{c o v} E$ is extreme, and hence is a face. 
In the proof of Theorem 1.4, we will need the following propositions:

Proposition 1.5. Let $Y$ be a compact convex set. Suppose $\mu, \nu \in M^{+}(Y)$ and $\mu \prec \nu$. Then supp $\mu \subseteq \overline{\operatorname{cov}}(\operatorname{supp} \nu)$.

Proof. Suppose $y \in \operatorname{supp} \mu-\overline{\operatorname{cov}}(\operatorname{supp} \nu)$. Then, there is a continuous affine function $u$ on $X$ such that $u(y)=-1$ and inf $u \overline{\overline{\operatorname{cov}}}$ $(\operatorname{supp} \nu))=0$. Let $w=\min \{u, 0\}$. Then $w$ is concave. Hence, $\int w d \nu \leqq \int w d \mu<0$. But, $w=0$ on supp $\nu-a$ contradiction.

Ppoposition 1.6. Let $F$ be a closed extreme set of the compact convex set $Y$. Suppose $\nu_{1} \prec \nu_{2}$ and supp $\nu_{1} \subseteq F$. Then supp $\nu_{2} \subseteq F$.

Proof. Let $J_{F}$ denote the function which is 0 on $F$ and 1 on $Y-F$. Then $J_{F}$ is 1.s.c. and concave. Let $D$ be the set of continuous concave functions on $Y$ which are strictly dominated (pointwise) by $J_{F}$. By a result of Mokobodski [11, p. 222], $D$ is directed upward and $J_{F}=\sup \{g \mid g \in D\}$. Since $\int g d \nu_{2} \leqq \int g d \nu_{1}$ for each $g \in D$, it follows that $\int J_{F} d \nu_{2} \leqq \int J_{F} d \nu_{1}$. (For a proof that

$$
\int J_{F} d \nu_{2}=\sup \left\{\int g d \nu_{2} \mid g \in D\right\},
$$

see, e.g., [4. p. 8].) Thus, $\nu_{2}(Y-F)=\nu_{1}(Y-F)=0$.

Proof of Theorem 1.4. Let $x \in \overline{\mathrm{cov}} E$. It will be shown that $\operatorname{supp} \mu \subseteq \overline{\operatorname{cov}} E$ for all $\mu \in R_{x}^{x}$. By [12, Lemma 4.1] and proposition 1.5, it is enough to prove that $\overline{\mathrm{cov}} E$ contains the support of every $\prec$-maximal measure in $R_{x}^{X}$. Since $X$ has the equal support property, it is only necessary to find one $\prec$-maximal representing measure which is supported by $E$. Let $\nu \in R_{x}^{E}$ (see [12, p. 5]). There is a $\prec$-maximal measure $\mu$ such that $\nu \prec \mu$ [12, Lemma 4.1]. By proposition 1.6 , it follows that $\operatorname{supp} \mu \subseteq E$.

In [1], Alfsen proved that if $Z$ is an $r$-simplex, i.e., a simplex whose set of extreme points is closed, then $\overline{\operatorname{cov}} B$ is a face for every $B \subseteq e x Z$. We will show that $X$ satisfies the conclusion of Alfsen's result if and only if $X$ has the equal support property and ex $X$ is closed. We claim that $X$ has the equal support property and $e x X=\partial X$ if and only if for each $x \in X$, all measures in $R_{x}^{\partial x}$ have the same 
support. The "only if" part of the previous statement follows from the fact that if supp $\mu \subseteq e x X$, then $\mu$ is $\prec-m a x i m a l ~[12, p p . ~ 26-27]$. Suppose that $z \in X$ and that all measures in $R_{z}^{\partial X}$ have the same support. Let $\nu$ be a $\prec$-maximal measure with $\varepsilon_{z} \prec \nu$. Since all $\prec$-maximal measures are supported by $X[12, \mathrm{p}$. 30], it follows that $\varepsilon_{z}=\nu$. Thus, $z \in e x X[12$, p. 27]. The proof of the above statement is complete. $\left(\varepsilon_{z}=\right.$ the Dirac measure at $z$.)

Definition 1.7. $X$ has the strong equal support property if, for each $x \in X$, all measures in $R_{x}^{\partial x}$ have the same support.

For the sake of brevity, we will use the abbreviation "s.e.s.p." to indicate the "strong equal support property".

THEOREM 1.8. The following are equivalent:

(i) $X$ has the s.e.s.p.

(ii) $\overline{\operatorname{cov}} B$ is extreme for every $B \cong \partial X$.

Proof. That (i) implies (ii) follows from Theorem 1.4 .

If (ii) holds, then $\operatorname{cov}\{x\}=\{x\}$ is extreme for each $x \in X$. Thus, $\partial X \cong e x X$. It must be shown that $X$ has the equal support property. Let $y \in X$ and let $\mu$ and $\nu$ be $\prec$-maximal representing measures for $y$. Since $y \in \overline{\operatorname{cov}}(\operatorname{supp} \mu)$, it follows, by Proposition 1.2, that supp $\nu \subseteq \overline{\operatorname{cov}}(\operatorname{supp} \mu)$. By $M T$ and Proposition 1.3, supp $\nu \cong \overline{\operatorname{cov}}$ $(\operatorname{supp} \mu) \cap e x X=\operatorname{supp} \mu$. Similarly, $\operatorname{supp} \mu \subseteq \operatorname{supp} \nu$.

In [8] Feinberg gave a proof of Theorem 1.8 which is independent of Theorem 1.4.

We are now able to give an example of a compact convex set which has the s.e.s.p., but is not a simplex.

EXAMPLE 1.9. Let $M([0,1])$ denote the space of real valued regular Borel measures on $[0,1]$. Assume that $M([0,1])$ is equipped with the weak* topology (recall that $M([0,1])$ is the dual of the space of real valued continuous functions on $[0,1])$. In [9, Remark 4] Lazar considers the quotient space $M([0,1]) / V$, where $V$ is the linear subspace of $M([0,1])$ spanned by a certain measure $\mu . \mu$ possesses the following properties: $\left.(\mathrm{P} 1) \mu^{\prime}[0,1]\right)=0$ and (P2) every open subinterval of $[0,1]$ contains sets of positive $\mu$-measure and sets of negative $\mu$-measure. Let $T$ be the restriction to $P([0,1]) \quad(P([0,1])=$ the probability measures on $[0,1])$ of the quotient map of $M([0,1])$ onto $M([0,1]) / V$ and let $Z=T(P([0,1]))$. Then $T$ maps ex $P([0,1])$ homeomorphically onto ex $Z$ and satisfies: $T^{-1}(T(F))=F$ for each $F \in \mathscr{F}(P([0,1]))$. Furthermore, $Z$ is not a simplex. 
It is claimed that $Z$ has the s.e.s.p. By Theorem 1.8 , it is enough to show that $\overline{\mathrm{cov}} E \in \mathscr{F}(Z)$ whenever $E$ is a closed subset of ex $Z$. Let $E^{\prime}=e x P([0,1]) \cap T^{-1}(E)$. Then, $T\left(\overline{\operatorname{cov}} E^{\prime}\right)=\overline{\operatorname{cov}} E$. By Theorem 1.8, it follows that $\overline{\operatorname{cov}} E^{\prime}$ is a face. Thus,

$$
T^{-1}(\overline{\operatorname{cov}} E)=\overline{\operatorname{cov}} E^{\prime} \text {. }
$$

A straightforward argument shows that $\overline{\operatorname{cov}} E$ is a face.

Rogalski [13, Prop. 13] has given an example of a compact convex set $Y$ which does not have the equal support property but which satisfies the following: If $E$ is a closed subset of $Y$ with $E \cong e x Y$, then $\overline{\operatorname{cov}} E$ is a face of $Y$.

2. Extremally concave functions. In this section $X$ will denote a compact convex set and $A$ will denote the set of real valued continuous affine functions on $X$. Consider the following abstract "Dirichlet" problem: (D) Given $f \in C(\partial X)(C(\partial X)=$ space of real continuous functions on $\partial X$ ), find $a_{f} \in A$ such that $a_{f} \mid \partial X=f$. In [2] Bauer characterized $r$-simplexes as follows: $X$ is an $r$-simplex if and only if (D) is solvable for each $f \in C(\partial X)$. In view of the definition of the equal support property, it is natural to expect that there is a characterization of compact convex sets with the s.e.s.p. which is similar to Bauer's result. In $\S 4$ we will characterize the s.e.s.p. in terms of a problem which is the same as (D) except that $A$ is replaced by a certain collection $\mathscr{E}(X)$ of functions on $X$. This section is concerned with investigating $\mathscr{E}(X)$.

Suppose $X$ has the s.e.s.p. Let $f \in C(e x X)$. For each $c \in(-\infty, \infty]$, let $F_{c}=\overline{\operatorname{cov}}\left(f^{-1}(-\infty, c]\right)$. By Theorem 1.8, $F_{c} \in \mathscr{F}(X)$ for each $c \in(-\infty, \infty]$. Define a function $f^{*}: X \rightarrow(-\infty, \infty]$ as follows: for each $x \in X$, let $f^{*}(x)=\inf \left\{c \mid x \in F_{c}\right\}$. Then $f^{*}$ extends $f$ and satisfies: $\left(f^{*}\right)^{-1}(-\infty, d] \in \mathscr{F}(X)$ for each $d \in(-\infty, \infty]$.

Definition 2.1. A function $g: X \rightarrow(-\infty, \infty]$ will be called extremally concave if $g^{-1}(-\infty, c] \in F(X)$ for each $c \in(-\infty, \infty]$. The set of all extremally concave functions on $X$ will be denoted by $\mathscr{f}(X)$.

Proposition 2.2. (a) Extremally concave functions are concave and 1.s.c. (b) For each $x \in X$ and each $\mu \in R_{x}^{x}, \int g d \mu \leqq g(x)$ for every $g \in \mathscr{E}(X)$.

Proof. Let $g$ be an extremally concave function. That $g$ is 
1.s.c. is clear. The fact that $g$ is concave is a special case of (b). Suppose $\mu \in R_{x}^{X}$. Let $\lambda=g^{\prime}(x)$. Since $x \in g^{-1}(\infty, \lambda]$, it follows that supp $\mu \leqq g^{-1}(-\infty, \lambda]$ (Prop. 1.2). Thus $\int g d \mu \leqq \lambda \mu(X)=g(x)$.

Proposition 2.3. Suppose $f, g \in \mathscr{C}(X)$. Then: (a) $f \leqq g$ if and only if $f|e x X \leqq g| e x X$. (b) $-f \leqq g$ if and only if $(-f)|e x X \leqq g| e x X$. It follows from (a) that extremally concave functions are uniquely determined by their values on ex $X$.

Proof. Suppose $f|e x X \leqq g| e x X$. Let $x \in X$ and let $b=g(x)$. Clearly, $\quad g^{-1}(-\infty, b] \cap e x X \cong f^{-1}(-\infty, b] \cap e x X$. By Proposition 1.3 and the KMT, $g^{-1}(-\infty, b] \subseteq f^{-1}(-\infty, b]$. Thus, $f(x) \leqq b=g(x)$.

Suppose $-f|e x X \leqq g| e x X$ and $(f+g)^{-1}(-\infty, 0) \neq \varnothing$. Then there are real numbers $s, t$ with $s<t$ and

$$
g^{-1}(-\infty, s] \cap(-f)^{-1}[t, \infty) \neq \varnothing .
$$

Since $g^{-1}(-\infty, s] \cap(-f)^{-1}[t, \infty) \in \mathscr{F}(X)$, it follows by Proposition 1.3 and the KMT that $g^{-1}(-\infty, s] \cap(-f)^{-1}[t, \infty) \cap e x X \neq \varnothing-$ a contradiction.

The next theorem is crucial in the proofs of some later theorems.

Theorem 2.4. Let $\mathscr{D} \subseteq \mathscr{E}(X)$. Then $\sup \{g \mid g \in \mathscr{D}\}$ (pointwise) is in $\mathscr{E}(X)$. Consequently, $\mathscr{E}(X)$ is a complete lattice in the pointwise ordering.

Proof. Let $k=\sup \{g \mid g \in D\}$. Suppose $c \in(-\infty, \infty]$. Then $k^{-1}(-\infty, c]=\cap\left\{g^{-1}(-\infty, c] \mid g \in D\right\}$. Since $\mathscr{F}(X)$ is closed under arbitrary intersections, it follows that $k \in \mathscr{E}(X)$.

Let $f, g \in \mathscr{E}(X) . \quad f \wedge g$ will denote $\sup \{k \mid k \in \mathscr{E}(X)$ and $k \leqq \min$ $\{f, g\}\}$.

We will complete this section by giving another property of extremally concave functions and some simple examples.

Proposition 2.5. Let $g \in \mathscr{E}(X), \quad x \in X$, and $\mu \in R_{x}^{X}$. Then $g(x)=\sup g(\operatorname{supp} \mu)$. In particular, if $x=c u+(1-c) v$ where $u, v \in X$ and $c \in(0,1)$, then $g(x)=\max \{g(u), g(v)\}$.

Proof. Since $x \in g^{-1}(-\infty, g(x)]$, it follows from Proposition 1.2 that $\operatorname{supp} \mu \subseteq g^{-1}(-\infty, g(x)]$. Thus, $\sup g(\operatorname{supp} \mu) \leqq g(x)$. Let $Z=\overline{\operatorname{cov}}$ $(\operatorname{supp} \mu$ ). Then $x \in Z$. Note that $g \mid Z \in \mathscr{E}(Z)$. (This fact follows from the fact that if $S$ is an extreme subset of $X$ then $S \cap Z$ is an extreme 
subset of $Z$.) By $M T$, ex $Z \cong \operatorname{supp} \mu$. By Proposition $2.3, g \mid Z \leqq \sup$ $g(\operatorname{supp} \mu)$.

The "In particular..." part of the proposition follows from the fact that $c \varepsilon_{u}+(1-c) \varepsilon_{v} \in R_{x}^{X}$.

Examples. Let $X=[0,1]$. Then $f \in \mathscr{E}(X)$ if and only if $f$ is in one of the following forms: (i) $f$ is a constant $b \in(-\infty, \infty]$ on $(0,1]$ and $f(0) \leqq b$. (ii) $f(x)=g(1-x)$ where $g$ is of form (i).

Let $X=\{(a, b) \mid a, b \in[0,1]$ and $a+b \leqq 1\}$. Let $F_{1}, F_{2}$, and $F_{3}$ be the "edges" of $X$. We will regard the $F_{i}$ 's as copies of $[0,1]$. Then $f \in \mathscr{E}(X)$ if and only if $f$ is in one of the forms $f(x)=b \in(-\infty, \infty]$ for $x \in X-F_{j}$ and $f(x)=g(x) \leqq b$, where $g \in \mathscr{E}\left(F_{j}\right)$, when $x \in F_{j}$ $j=1,2,3$.

3. The structure topology, Let $Z$ be a simplex. Effros [6, p. 117] has defined a topology for ex $Z$ called the structure topology . In this section, we extend Effros' definition to a larger class of compact convex sets. Alfsen and Andersen [2] have defined a topology called the facial topology, for the set of extreme points of an arbitrary compact convex set. The facial topology is a generalization of Effros' structure topology. At the end of this section, we will make a comparison between the structure and facial topologies.

For the rest of this section, $X$ will denote a nonempty compact convex set. Let $\mathscr{T}_{X}=\{F \cap e x X \mid F \in \mathscr{F}(X)\}$. Note that $\varnothing$, ex $X \in \mathscr{T}_{X}$ and that the intersection of any sub-collection of $\mathscr{T}_{X}$ is in $\mathscr{T}_{X}$. Suppose $X$ has the e.s.p. Consider $F, F^{\prime} \in \mathscr{F}(X)$. By Theorem 1.4, $\operatorname{cov}\left(F \cup F^{\prime}\right) \in \mathscr{F}(X)$. By Proposition 1.3,

$$
(F \cap e x \quad X) \cup\left(F^{\prime} \cap e x \quad X\right)=e x X \cap \operatorname{cov}\left(F \cup F^{\prime}\right) .
$$

It follows that the union of any finite sub-collection of $\mathscr{T}_{X}$ is in $\mathscr{T}_{X}$. Thus, $\mathscr{T}_{X}$ is the collection of closed sets for a topology on ex $X$, whenever $X$ has the e.s.p.

Definition 3.1. If $\mathscr{T}_{X}$ is closed under finite unions, then the topology on $e x X$ for which $\mathscr{T}_{X}$ is the collection of closed sets will be called the structure topology. We will use the adjective "structurally" to replace "... in the structure topology", e.g., "structurally compact" means "compact in the structure topology".

THEOREM 3.2. The following are equivalent:

(i) The structure topology exists on ex $X$.

(ii) $\operatorname{cov}\left(F \cup F^{\prime}\right) \in \mathscr{F}(X)$ for all $F, F^{\prime} \in \mathscr{F}(X)$.

(iii) $\operatorname{cov}\left(F \cup F^{\prime}\right)=F \vee F^{\prime}$ for all $F, F^{\prime} \in(X)$.

(iv) $\mathscr{F}(X)$ is a distributive lattice. 
Proof. The equivalence of (i) and (ii) follows from Proposition 1.3. The equivalence of (ii) and (iii) is clear.

Let $F, F^{\prime \prime} \in \mathscr{F}(X)$. Suppose (iii) holds. To show that

$$
F \cap\left(F^{\prime} \vee F^{\prime \prime}\right)=\left(F \cap F^{\prime \prime}\right) \vee\left(F \cap F^{\prime \prime}\right),
$$

it is enough to prove that

$$
F \cap \operatorname{cov}\left(F^{\prime \prime} \cup F^{\prime \prime}\right)=\operatorname{cov}\left(\left(F \cap F^{\prime}\right) \cup\left(F \cap F^{\prime \prime}\right)\right) .
$$

By the $K M T$, equation (1) is equivalent to

$$
e x\left(F \cap \operatorname{cov}\left(F^{\prime} \cup F^{\prime \prime}\right)\right)=e x\left(\operatorname{cov}\left(\left(F \cap F^{\prime}\right) \cup\left(F \cap F^{\prime \prime}\right)\right)\right) .
$$

Equation (2) follows from Proposition 1.3. Suppose (iv) holds and $F \vee F^{\prime}$ properly contains cov $\left(F \cup F^{\prime}\right)$. By the $K M T$ and Proposition 1.3, there is a point $x_{1} \in e x \quad X \cap\left(E \vee F^{\prime}-\operatorname{cov}\left(F \cup F^{\prime}\right)\right)$. Since $\left\{x_{1}\right\} \in \mathscr{F}(X)$ we have

$$
\begin{aligned}
\left\{x_{1}\right\} & =\left\{x_{1}\right\} \cap\left(F \vee F^{\prime}\right) \\
& =\left(\left\{x_{1}\right\} \cap F\right) \vee\left(\left\{x_{1}\right\} \cap F^{\prime \prime}\right) \\
& =\varnothing \vee \varnothing=\varnothing .
\end{aligned}
$$

The next lemma indicates that compact convex sets $X$ for which $\mathscr{F}(X)$ is distributive are similar to simplexes.

Lemma 3.3. Suppose $\mathscr{F}(X)$ is distributive. Let $S \subseteq$ ex $X$. Then cov $S$ is extreme and each point in cov $S$ has a unique <-maximal representing measure.

Proof. To prove that cov $S$ is extreme, it is enough to consider the case where $S$ is finite. cov $S$ is surely extreme if $S$ is a singleton. Suppose that, for sets $S$ of cardinality $<$ the integer $n$, cov $S$ is extreme. Let $B$ be a subset of $e x X$ with $n$ elements. Choose some $y \in B$. Then $\operatorname{cov} B=\operatorname{cov}(\{y\} \cup \operatorname{cov}(B-\{y\}))$. By Theorem 3.2, $\operatorname{cov} B$ is extreme.

We will now prove the second assertion. Let $z \in \operatorname{cov} S$. Then $z$ may be written in the form $(\dagger) z=\sum_{i=1}^{p} c_{i} x_{i}$ where the $x_{i}$ 's are distinct elements of $S, c_{i}>0$ for $i=1,2 \cdots, p$ and $\sum_{i=1}^{p} c_{i}=1$. By Lemma 1.2, all representing measures for $z$ are supported by cov $\left\{x_{1}, \cdots, x_{p}\right\}$. Thus, to show that $\sum_{i=1}^{p} c_{i} \varepsilon_{x_{i}}$ is the only maximal representing measure for $z$, it is enough to show that the representation $(\dagger)$ is unique (see [18, p. 26]). Let $z=\sum_{j=1}^{q} b_{j} y_{j}$ be another representation for $z$ of the form $(\dagger)$. Since $\operatorname{cov}\left\{x_{1}, \cdots, x_{p}\right\}$ is extreme,

$$
\begin{aligned}
\left\{y_{1}, \cdots, y_{q}\right\} & \leqq \operatorname{cov}\left\{x_{1}, \cdots, x_{p}\right\} \cap e x X \\
& \cong\left\{x_{1}, \cdots, x_{p}\right\} .
\end{aligned}
$$


Similarly, $\left\{x_{1}, \cdots, x_{p}\right\} \subseteq\left\{y_{1}, \cdots, y_{q}\right\}$. Assume $y_{i}=x_{i} i=1,2, \cdots, p$. Suppose $b_{1}>c_{1}$. Then

$$
\left(\frac{b_{1}-c_{1}}{1-c_{1}}\right) x_{1}+\frac{b_{2}}{1-c_{1}} x_{2}+\cdots+\frac{b_{p}}{1-c_{1}} x_{p}
$$

$\in \operatorname{cov}\left\{x_{2}, \cdots, x_{p}\right\}$. Thus $x_{1} \in \operatorname{cov}\left\{x_{2}, \cdots, x_{p}\right\}-$ a contradiction. Hence, $c_{1} \geqq b_{1}$. Similarly, $b_{1} \geqq c_{1}$. By the same argument, $c_{i}=b_{i}, i=2, \cdots, p$.

Theorem 3.4. Suppose $X$ is a compact convex subset of a finite dimensional linear space. Then the following are equivalent:

(i) For some integer $n$, there is an affine homeomorphism of $X$ onto the standard n-simplex.

(ii) $X$ is a simplex.

(iii) $\mathscr{F}(X)$ is distributive.

(iv) $X$ has the e.s.p.

Proof. For a proof of the equivalence of (i) and (ii) see [18, Prop. 9. 11]. At the beginning of this section we proved that (iv) implies (iii), and it is clear that (ii) implies (iv). Suppose (iii) holds. By a theorem of Minkowski [18, p. 1], $X=\operatorname{cov}(e x X)$. By the previous lemma, $X$ is a simplex.

In [10], Feinberg proved the equivalence of (ii) and (iii). Theorem 3.4 shows that compact convex sets $X$ for which $\mathscr{F}(X)$ is distributive and those which have the equal support property are generalizations of finite dimensional simplexes. The following example shows that they are distinct generalizations:

EXAMPle 3.5. The technique used in constructing this example is due to Alfsen [1]. Let $N^{*}$ be the one-point compactification of the space of positive integers. Then $C\left(N^{*}\right)$ is the space of real sequences of the form $\left(a_{1}, a_{2} \cdots ; a_{\infty}\right)$ where $a_{\infty}=\lim a_{n}$. Let

$$
M\left(N^{*}\right)=M^{+}\left(N^{*}\right)-M^{+}\left(N^{*}\right),
$$

then $M\left(N^{*}\right)$ is the dual space of $C\left(N^{*}\right)$ and may be identified with the space of real sequences of the form $\left(x_{1}, x_{2}, \cdots ; x_{\infty}\right)$ where $\sum_{i=1}^{\infty}\left|x_{i}\right|<\infty$. It will be assumed that $M\left(N^{*}\right)$ is equipped with the weak* topology. $P\left(N^{*}\right)$ will denote $\left\{\left(x_{1}, \cdots ; x_{\infty}\right) \mid x_{n} \geqq 0 n=1,2, \cdots \infty\right.$ and $\left.x_{\infty}+\sum_{n=1}^{\infty} x_{n}=1\right\}$. Then $P\left(N^{*}\right)$ is a compact convex subset of $M\left(N^{*}\right)$, indeed $P\left(N^{*}\right)$ is a simplex (see, e.g., [2, Satz 13]). The extreme points of $P\left(N^{*}\right)$ are those of the form $\varepsilon_{p}$ where $\varepsilon_{p}$ is 1 at the $p$-th and 0 at every other position $p=1,2, \cdots, \infty$ (see $[4, \mathrm{p}$. 441]). 
Consider the sequences $u=(1 / 2,0,1 / 4,0,1 / 8,0, \cdots-1)$ and $v=(0,1 / 2,0,1 / 4,0,1 / 8, \cdots-1)$. Let $L$ be the subspace of $M\left(N^{*}\right)$ generated by $u$ and $v$. Consider the quotient space $V=M\left(N^{*}\right) / L$ and let $T$ be the cannonical projection of $M\left(N^{*}\right)$ onto $V$. Let $Z=T\left(P\left(N^{*}\right)\right)$. It is claimed that ex $Z=\left\{T\left(\varepsilon_{n}\right) \mid 1 \leqq n<\infty\right\}$. Suppose $y \in e x Z$. Then $T^{-1}(y) \cap P\left(N^{*}\right)$ is a closed convex extreme subset of $P\left(N^{*}\right)$. By Proposition 1.3, and the $K M T$, it follows that $y=T\left(\varepsilon_{n}\right)$ for some $1 \leqq n \leqq \infty$. It must be shown that $n \neq \infty$. Let $\delta_{n}$ denote the Dirac measure at $T\left(\varepsilon_{n}\right)$ for $n=1,2, \cdots, \infty$. The series $\sum_{j=1}^{\infty}$ $1 / 2^{j} \delta_{2 j-1}$ converges in the norm topology of the dual of $C(Z)$ to a measure $\mu_{1}$ which is $\prec$-maximal and represents $T\left(\varepsilon_{\infty}\right)$ (see [18, p. 26]). It follows that $T\left(\varepsilon_{\infty}\right) \notin e x Z$.

Next, it is asserted that $F \in \mathscr{F}(Z)$ if and only if $F=X$ or $F=\operatorname{cov} E$ where $E$ is a finite subset of ex $Z$. Suppose $F \in \mathscr{F}(Z)$ and $e x F$ is infinite. Then $T^{-1}(F) \cap e x P\left(N^{*}\right)$ is infinite. It follows that $\varepsilon_{\infty} \in T^{-1}(F)$. The measure $\mu_{2}=\sum_{i=1}^{\infty} 1 / 2^{j} \delta_{2 j-2}$ is a $\prec$-maximal representing measure for $T\left(\varepsilon_{\infty}\right)$. By Lemma 1.2, supp $\mu_{2} \cup \operatorname{supp} \mu_{1} \subseteq F$. But supp $\mu_{1} \cup \operatorname{supp} \mu_{2} \supseteqq e x Z$. By the $K M T$, it follows that $F=Z$. Conversely, suppose $F=\operatorname{cov}\left\{x_{1}, \cdots, x_{n}\right\}$ where $\left\{x_{1}, \cdots, x_{n}\right\} \subseteq$ ex $Z$. To prove $F \in \mathscr{F}(Z)$, it is enough to show $T^{-1}(F) \cap P\left(N^{*}\right) \in \mathscr{F}\left(P\left(N^{*}\right)\right)$. There are integers $k_{1}, k_{2}, \cdots, k_{n}$ such that $T\left(\varepsilon_{k_{j}}\right)=x_{j}, j=1,2, \cdots, n$. Clearly, $\operatorname{cov}\left\{\varepsilon_{k_{1}}, \cdots, \varepsilon_{k_{n}}\right\} \subseteq T^{-1}(F) \cap P\left(N^{*}\right)$ and $T\left(\operatorname{cov}\left\{\varepsilon_{k_{1}}, \cdots, \varepsilon_{k_{n}}\right\}\right)=F$. It follows that, if $w=\left(a_{1}, \cdots ; a_{\infty}\right) \in T^{-1}(F) \cap P\left(N^{*}\right)$, then there is an $s \in \operatorname{cov}\left\{\varepsilon_{k_{1}}, \cdots, \varepsilon_{k_{n}}\right\}$ such that $w-s=c u+d v$ where $c, d$ are real numbers. Hence, $a_{\infty}=-(c+d) \geqq 0$. Also, there integers $p$ and $q$ such that $a_{2 p-1}=1 / 2^{p} c$ and $a_{2 q}=1 / 2^{q} d$. Thus, $c=d=0$. It follows that $\operatorname{cov}\left\{\varepsilon_{k_{1}}, \cdots, \varepsilon_{k_{n}}\right\}=T^{-1}(F) \cap P\left(N^{*}\right)$.

It is now clear that $\operatorname{cov}(F \cup E) \in \mathscr{F}(Z)$ for all $F, E \in \mathscr{F}(Z)$. By Theorem 3.2, $\mathscr{F}(X)$ is distributive. $Z$ does not, however, have the e.s.p. for the measures $\mu_{1}$ and $\mu_{2}$ are both $\prec$-maximal and represent $T\left(\varepsilon_{\infty}\right)$ but $\operatorname{supp} \mu_{1} \neq \operatorname{supp} \mu_{2}$.

It is interesting to note that the structure topology on ex $Z$ is the smallest $T_{1}$ topology on ex $Z$.

The following theorem extends [7, Corollary 4.6].

Theorem 3.6. If the structure topology is defined on ex $X$, then ex $X$ is structurally compact.

Proof. Let $\mathscr{J}$ be a collection of structurally closed subsets of $e x X$ having the finite intersection property. For each $C \in \mathcal{J}$, there is a $F_{C} \in \mathscr{F}(X)$ such that $F_{C} \cap$ ex $X=C$. Hence, the family $\left\{F_{C} \mid C \in \mathscr{J}\right\}$ has the finite intersection property. Thus, $\cap\left\{F_{C} \mid C \in \mathscr{J}\right\}$ is nonempty. By the $K M T$ and Proposition 1.3, it follows that $\cap\{C \mid C \in \mathscr{J}\} \neq \varnothing$. 
THEOREM 3.7. Suppose the structure topology is defined on ex $X$. Let $f: e x \quad X \rightarrow(-\infty, \infty]$ be structurally 1.s.c., then there is an $f^{*} \in \mathscr{E}(X)$ such that $f^{*} \mid$ ex $X=f$.

Proof. For each $b \in(-\infty, \infty]$, let $F_{b}=\overline{\operatorname{cov}} f^{-1}(-\infty, b]$. By Proposition 1.3, and the KMT, it follows that $F_{b} \in \mathscr{F}(X)$ for all $b \in(-\infty, \infty]$. Define $f^{*}$ by: $f^{*}(x)=\inf \left\{b \mid x \in F_{b}\right\}$.

Notation. Suppose $f: e x X \rightarrow(-\infty, \infty]([-\infty, \infty))$. If there is an extension of $f$ to $\mathscr{E}(X)(-\mathscr{E}(X))$, denote it by $f^{*}\left(f_{*}\right)$.

When the structure topology is defined, it is stronger than the facial topology. If the facial topology is Hausdorff, then the facial, structure, and relative topologies coincide (see [2, Th. 6.2]).

Consider Example 1.9. We will identify ex $P([0,1])$ and $[0,1]$ and regard each $\rho \in P([0,1])$ as its own unique $\prec$-maximal representing measure. Note that, on ex $Z$, "closed" and "structurally closed" mean the same thing. Let $E$ be a proper closed subset of $e x Z$ and let $E^{\prime}=[0,1] \cap T^{-1}(E)$. It is known that the set $F=\overline{\operatorname{cov}} E$ is a face of $Z$. Furthermore, $T^{-1}(F)=\operatorname{cov} E^{\prime}=\left\{\rho \mid \operatorname{supp} \rho \subseteq E^{\prime}\right\}$ is a face of $P([0,1])$. We claim that $E$ is closed in the facial topology if and only if $|\mu|\left(E^{\prime}\right)=0$, where $|\mu|$ denotes the total variation of $\mu$. (It will be convenient to assume that $|\mu|([0,1])=2$.)

Suppose that $|\mu|\left(E^{\prime}\right)=0$. It is enough to show that $F$ is a split face ([2, p. 9]). Let $G=\left\{x \mid x=T(\nu)\right.$ where $\nu\left(E^{\prime}\right)=0$. Then $G$ is a face of $Z$ and, since $T^{-1}(F)=\overline{\operatorname{cov}} E^{\prime}, G$ is disjoint from $F$. Since every $\rho \in P([0,1])$ can be written as a convex combination of a measure in $\overline{\mathrm{cov}} E^{\prime}$ and a measure in $P([0,1])$ which vanishes on $E^{\prime}$, it follows that $Z=\operatorname{cov}(F \cup G)$. Thus, any face of $Z$ which is disjoint from $F$ must be contained in $G$. Hence, $G$ is the complementary set ([2]) of $F$. Suppose that $w \in Z-F \cup G$ and

$$
w=c_{1} u_{1}+\left(1-c_{1}\right) v_{1}=c_{2} u_{2}+\left(1-c_{2}\right) v_{2},
$$

where $0<c_{i}<1, u_{i} \in F$, and $v_{i} \in G$ for $i=1,2$. For $i=1,2$ choose $\alpha_{i}$ such that $T\left(\alpha_{i}\right)=u_{i}$, and $\beta_{i}$ such that $\beta_{i}\left(E^{\prime}\right)=0$ and $T\left(\beta_{i}\right)=v_{i}$. Then there is a real number $k$ such that

$$
c_{1} \alpha_{1}+\left(1-c_{1}\right) \beta_{1}-c_{2} \alpha_{2}-\left(1-c_{2}\right) \beta_{2}=k \mu \mu .
$$

Since $|\mu|\left(E^{\prime}\right)=0$, it follows that $c_{1}=c_{2}$ and $\alpha_{1}=\alpha_{2}$. Hence, $F$ is a split face.

Assume that $|\mu|\left(E^{\prime}\right)>0$. It will be shown that $F$ is not split.

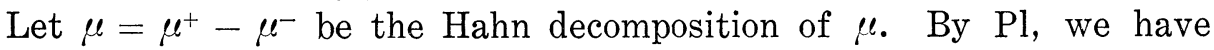
$\mu^{+}, \mu^{-} \in P(0,1)$.

Case 1. $\mu^{+}\left(E^{\prime}\right)>0$ and $\mu^{-}\left(E^{\prime}\right)>0$. By P2, we have $1>\mu^{+}(E)$, 
$\mu^{-}\left(E^{\prime}\right)$. Let $c_{1}=\mu^{+}\left(E^{\prime}\right)$ and $c_{2}=\mu^{-}\left(E^{\prime}\right)$. Define measures $\alpha_{1}, \alpha_{2}, \beta_{1}, \beta_{2}$ as follows: $\alpha_{1}(B)=c_{1}^{-1} \mu^{+}\left(E^{\prime} \cap B\right), \alpha_{2}(B)=c_{2}^{-1} \mu^{-}\left(E^{\prime} \cap B\right), \quad \beta_{1}(B)=$ $\left(1-c_{1}\right)^{-1} \mu^{+}\left(B-E^{\prime}\right)$, and $\beta_{2}(B)=\left(1-c_{2}\right)^{-1} \mu^{-}\left(B-E^{\prime}\right)$. Then $c_{1} x_{1}+\left(1-c_{1}\right) y_{1}=$ $c_{2} x_{2}+\left(1-c_{2}\right) y_{2}$, where $x_{i}=T\left(\alpha_{i}\right)$ and $y_{i}=T\left(\beta_{i}\right)$ for $i=1,2$. A straightforward argument shows that either $c_{1} \neq c_{2}, x_{1} \neq x_{2}$, or $y_{1} \neq y_{2}$. Since $\alpha_{1}, \alpha_{2}$ are supported by $E^{\prime}$, it follows that $x_{1}, x_{2} \in F$. Suppose it can be shown that $y_{1}$ and $y_{2}$ are in the complementary set of $F$. Then, by definition, $F$ is not a split face.

The set $S=\left\{u \in Z \mid c u+(1-c) v=y_{1}\right.$ for some $\left.(c, v) \in(0,1) \times Z\right\}$. is the smallest face containing $y_{1}$. Suppose that cu $+(1-c) v=y_{1}$. Choose $\nu_{1}, \nu_{2}$ such that $u=T\left(\nu_{1}\right)$ and $v=T\left(\nu_{2}\right)$. Then there is a real number $k$ such that $c \nu_{1}+(1-c) \nu_{2}-\beta_{1}=k \mu$. It follows that $k \mu$ is non-negative on subsets of $E^{\prime}$. Thus, $k=0$. It follows that $u, v \notin F$. Hence, $S \cap F=\varnothing$. i.e., $y_{1}$ is in the complementary set of $F$. Similarly, $y_{2}$ is in the complementary set of $F$.

Case 2. $\mu^{+}\left(E^{\prime}\right)>0$ and $\mu^{-}\left(E^{\prime}\right)=0$. Let I denote the characteristic function of the set $F$. The pointwise inf of the set of all continuous affine functions strictly dominating $I$ will be denoted by $\hat{I}$. Suppose $F$ is a split face then, by [2, Th. 3.5], $\hat{I}$ is affine. Define the measure $\mu^{+} \circ T^{-1}$ by $\mu^{+} \circ T^{-1}(B)=\mu^{+}\left(T^{-1}(B)\right)$. Define $\mu^{-} \circ T^{-1}$ similarly. Then $\mu^{+} \circ T^{-1}$ and $\mu^{-} \circ T^{-1}$ are $\prec$-maximal representing measures for the point $T\left(\mu^{+}\right)=T\left(\mu^{-}\right)$. It follows by [12, Lemma 9.7] that

$$
\int \hat{I} d \mu^{+} \circ T^{-1}=\int \hat{I} d \mu^{-} \circ T^{-1} .
$$

Since $I$ is u.s.c. on $Z$, we have $I=\hat{I}$ on $e x Z$ (see [12, p. 27]). It follows that $\mu^{+} \circ T^{-1}(E)=\mu^{-} \circ T^{-1}(E)$. Therefore, $\mu^{+}\left(E^{\prime}\right)=\mu^{-}\left(E^{\prime}\right)=0-\mathbf{a}$ contradiction. Hence, $F$ is not split.

The case in which $\mu^{+}\left(E^{\prime}\right)=0$ and $\mu^{-}\left(E^{\prime}\right)>0$ is handled in the same way as Case 2.

By P2, a proper subset of ex $Z$ which is closed in the facial topology, is nowhere dense in the structure topology.

\section{Characterizations of the strong equal support property.}

THEOREM 4.1. Let $X$ be a nonempty compact convex set. Then the following are equivalent:

(i) $X$ has the s.e.s.p.

(ii) For each $f \in C(\partial X)$, there is a function $f^{*} \in \mathscr{E}(X)$ with $f^{*} \mid \partial X=f$

(iii) $\mathscr{E}(X)$ is distributive and separates the points of ex $X$ in the following sense: if $x$ and $y$ are distinct extreme points of $X$, then there are $g, f \in E(X)$ such that $f(x)>0, g(y)>0$ and $f \wedge g \leqq 0$ 
(iv) $\mathscr{F}(X)$ is distributive and ex $X$ is structurally Hausdorff.

(v) For each continuous affine function a on $X$, there is an $a^{*} \in \mathscr{E}(X)$ such that $a\left|e x X=a^{*}\right|$ ex $X$.

Proof. For a proof that (i) implies (ii), see the discussion preceding Definition 2.1.

(ii) $\rightarrow$ (iii). Suppose $x$ and $y$ are distinct elements of $e x X$. There are functions $h, k \in C(\partial X)$ such that $h(x)>0, k(y)>0$, and $\min \{h, k\} \leqq 0$. Since $h^{*}, k^{*} \mid e x X \leqq 0$, it follows by Proposition 2.3 that $h^{*} \wedge k^{*} \leqq 0$.

To prove that $\mathscr{E}(X)$ is distributive, it is enough to show that, for all $f, g \in \mathscr{E}(X)$,

$(\dagger) f \wedge g \mid \partial X=\min \{f, g\} ; \partial X$.

Since $f$ and $g$ are 1.s.c., it follows that $f \mid \partial X=\sup \{u|u<f| \partial X$ and $u \in C(\partial X)\}$ and $g \mid \partial X=\sup \{v|v<g| \partial X$ and $v \in C(\partial X)\}$. Let $h=\sup \left\{u^{*} \wedge v^{*}|u, v \in C(\partial X), u \leqq f| \partial X, v \leqq g \mid \partial X\right\}$. By Theorem 2.4, it follows that $h \in \mathscr{E}(X)$. Note that, if $u, v \in C(\partial X), u^{*} \wedge v^{*}=$ $(\min \{u, v\})^{*}$ (Prop. 2.3). Thus, $h \mid \partial X=\min \{f|\partial X, g| \partial X\}$. It follows by Proposition 2.3 that $h=f<g$.

(iii) $\rightarrow$ (iv). First, it will be shown that $\mathscr{F}(X)$ is distributive. We will use $J_{S}$ to denote the function which is 0 on $S$ and 1 elsewhere. Let $F, E \in \mathscr{F}(X)$. Then $J_{F}, J_{E} \in \mathscr{E}(X)$. Note that $J_{F} \wedge J_{E}=J_{F \vee E}$. Suppose $x \in((F \vee E) \cap e x X)-\operatorname{cov}(F \cup E)$. Then

$$
\begin{aligned}
J_{\{x\}} & =\max \left\{J_{\{x\}}, J_{F^{\prime}} \wedge J_{E}\right\} \\
& =\left(\max \left\{J_{\{x\}}, J_{F}\right\}\right) \wedge\left(\max \left\{J_{\{x\}}, J_{E}\right\}\right) \\
& =1 \wedge 1=1 .
\end{aligned}
$$

Thus, $((F \vee E) \cap e x X)-\operatorname{cov}(F \cup E)=\varnothing$. By Proposition 1.3 and the $K M T, F \vee E=\operatorname{cov}(F \cup E)$. It follows by Theorem 3.2 that $\mathscr{F}(X)$ is distributive.

To prove that $e x X$ is structurally Hausdorff, it is enough to show that, for each pair $x, y \in e x \quad X, x \neq y$, there are $F, E \in \mathscr{F}(X)$ such that $x \notin F, y \notin E$ and $F \cup E \supseteqq e x X$. By hypothesis, there are functions $f, g \in \mathscr{E}(X)$ such that $f(x)>0, g(y)>0$ and $f \wedge g \leqq 0$. Let $F=f^{-1}(-\infty, 0]$ and $E=g^{-1}(-\infty, 0]$. Since $\min \{f|e x X, g| e x X\}$ is structurally 1.s.c., it follows by Theorem 3.7 and Proposition 2.3 that $f \wedge g|e x X=\min \{f, g\}| e x X$. Thus, $(F \cup E) \cap e x X=e x X$.

(iv) $\rightarrow$ ( i ). Suppose it can be shown that ex $X$ is closed. Then, since the structure topology is weaker than the relative topology, every closed subset of $e x X$ is structurally closed. Thus, if $E \cong e x X$ is closed, then there is an $F \in \mathscr{F}(X)$ with $E=F \cap e x \quad X$. By Proposition 1.3, $\overline{\mathrm{cov}} E=F$. It follows by Theorem 1.8 that $X$ has 
the s.e.s.p.

It remains to be shown that $e x X$ is closed. Let $C_{s}(e x X)$ be the space of structurally continuous real-valued functions on $e x X$. Suppose $z_{1} \in \partial X$. Then there is a net $\left\{z_{\alpha}\right\}$ of elements in ex $X$ which converges to $z_{1}$ and converges structurally to some $z \in e x X$. Hence, by Theorem 3.7 and the proof of Theorem 3.8, $f^{*}\left(z_{1}\right)=f(z)$ for each $f \in C_{s}(e x X)$. Note that $J_{\{z\}} \mid e x X$ is structurally 1.s.c. Since ex $X$ is structurally compact and Hausdorif, it follows that

$$
J_{\{z\}} \mid \operatorname{ex} X=\sup \left\{f \in C_{s}(e x X)\left|f<J_{\{z\}}\right| \operatorname{ex} X\right\} \text {. }
$$

By Theorem 2.4 and Proposition 2.3, it follows that

$$
J_{\{z\}}=\sup \left\{f^{*}\left|f \in C_{s}(e x \quad X), f<J_{\{z\}}\right| e x X\right\} \text {. }
$$

Therefore, $J_{\{z\}}\left(z_{1}\right)=J_{\{2\}}(z)$. Thus, $z=z_{1}$.

That (ii) implies ( $\mathrm{v}$ ) is clear.

$(\mathrm{v}) \rightarrow(\mathrm{iv})$. First it will be shown that $\mathscr{F}(X)$ is distributive. Let $F, E \in \mathscr{F}(X)$. Let $K=\left\{a \mid a\right.$ is continuous affine and $a^{-1}(-\infty, 0] \supseteqq$ $\operatorname{cov}(F \cup E)\}$. It is claimed that

$$
\operatorname{cov}(F \cup E)=\cap\left\{\left(a^{*}\right)^{-1}(-\infty, 0] \mid \alpha \in K\right\} \text {. }
$$

If $a \in K$, then $\left(a^{*}\right)^{-1}(-\infty, 0] \supseteqq e x(\operatorname{cov}(F \cup E))$ by Proposition 1.3. Suppose $a^{*}(x) \leqq 0$ for every $a \in K$. If $h$ is any real continuous affine function on $X$, then $h^{*}-h$ is concave and 1.s.c. Since $\left(h^{*}-h\right) \mid e x X \geqq 0$, it follows that $h^{*} \geqq h$. Thus, $a(x) \leqq 0$ for every $a \in K$. A simple separation argument shows that $x \in \operatorname{cov}(F \cup E)$.

To prove that ex $X$ is structurally Hausdorff, it is enough to note that $C_{s}(e x X)$ contains a point-separating subspace.

Many of the results contained in this paper can be generalized to the following setting: Let $Y$ be a compact Hausdorff space. Suppose that $S$ is a cone of real-valued continuous functions on $Y$ which satisfies: $f, g \mathscr{S}$ implies that $\min \{f, g\} \in \mathscr{S}$. A notion of "convexity with respect to $\mathscr{S}$ " can be introduced on $Y$. The concepts of: extreme point, extreme set, face, and structure topology can be given meaning in the above context. For details, see [10].

\section{REFERENCES}

1. E. M. Alfsen, On the geometry of Choquet simplexes, Math. Scand., 15 (1964), 97-110.

2. E. M. Alfsen and T. B. Andersen, Split faces of compact convex sets, Aarhus Universitet, preprint series 1968/1969 No. 32 .

3. H. Bauer, Shilovscher Rand und Dirichletsches Problem, Ann. Inst. Fourier (Grenoble) 11 (1961), 89-136. 
4. M. Brelot, Elements de la theorie classique du potential, 3rd ed., Centre Documentation Univ., Paris, 1965.

5. N. Dunford and J. T. Schwartz, Linear Operators I, Interscience, New York, 1957.

6. E. Effros, Structure in simplexes, Acta. Math., 117 (1967), 103-121.

7. _- Structure in simplexes II, J. of Functional Analysis, 1 (4) (1967), 379-391.

8. I. Feinberg, Henry Rutgers thesis, Rutgers-The State University (1968).

9. A. J. Lazar, On affine functions on simplexes, Ditoed notes, Hebrew University, Jerusalem.

10. J. N. Mc Donald, Thesis, Rutgers-The State University (1969).

11. P. A. Meyer, Probability and potentials, Blaisdell, Waltham, Mass. (1966).

12. R. R. Phelps, Lectures on Choquet's theorem, Van Nostrand, Princeton, N. J. (1966).

13. M. Rogalski, Quelques problèmes concernant une caracterisation des simplexes,

C. R. Acad. Sci., Paris, Oct. (1969).

Received January 22, 1970. This paper is based on part of the author's Ph. D. thesis, written at Rutgers-The State University under Professor Marvin Grossman. Part of the research for this paper was done while the author was on an NSF Summer Traineeship. Part of the work presented here was supported by an Arizona State University Faculty Grant.

Arizona State University 



\section{PACIFIC JOURNAL OF MATHEMATICS}

\section{EDITORS}

\author{
H. SAMElson \\ Stanford University \\ Stanford, California 94305 \\ C. R. HOBBY \\ University of Washington \\ Seattle, Washington 98105
}

J. DugundjI

Department of Mathematics University of Southern California Los Angeles, California 90007

RICHARD ARENS

University of California

Los Angeles, California 90024

\section{ASSOCIATE EDITORS}
E. F. BECKENBACH
B. H. NeUmanN
F. WOLE
K. YOSHIDA

\section{SUPPORTING INSTITUTIONS}

\author{
UNIVERSITY OF BRITISH COLUMBIA \\ CALIFORNIA INSTITUTE OF TECHNOLOGY \\ UNIVERSITY OF CALIFORNIA \\ MONTANA STATE UNIVERSITY \\ UNIVERSITY OF NEVADA \\ NEW MEXICO STATE UNIVERSITY \\ OREGON STATE UNIVERSITY \\ UNIVERSITY OF OREGON \\ OSAKA UNIVERSITY \\ UNIVERSITY OF SOUTHERN CALIFORNIA
}

\author{
STANFORD UNIVERSITY \\ UNIVERSITY OF TOKYO \\ UNIVERSITY OF UTAH \\ WASHINGTON STATE UNIVERSITY \\ UNIVERSITY OF WASHINGTON \\ * * * \\ AMERICAN MATHEMATICAL SOCIETY \\ CHEVRON RESEARCH CORPORATION \\ NAVAL WEAPONS CENTER
}

The Supporting Institutions listed above contribute to the cost of publication of this Journal, but they are not owners or publishers and have no responsibility for its content or policies.

Mathematical papers intended for publication in the Pacific Journal of Mathematics should be in typed form or offset-reproduced, (not dittoed), double spaced with large margins. Underline Greek letters in red, German in green, and script in blue. The first paragraph or two must be capable of being used separately as a synopsis of the entire paper. The editorial "we" must not be used in the synopsis, and items of the bibliography should not be cited there unless absolutely necessary, in which case they must be identified by author and Journal, rather than by item number. Manuscripts, in duplicate if possible, may be sent to any one of the four editors. Please classify according to the scheme of Math. Rev. Index to Vol. 39. All other communications to the editors should be addressed to the managing editor, Richard Arens, University of California, Los Angeles, California, 90024.

50 reprints are provided free for each article; additional copies may be obtained at cost in multiples of 50 .

The Pacific Journal of Mathematics is published monthly. Effective with Volume 16 the price per volume (3 numbers) is $\$ 8.00$; single issues, $\$ 3.00$. Special price for current issues to individual faculty members of supporting institutions and to individual members of the American Mathematical Society: $\$ 4.00$ per volume; single issues $\$ 1.50$. Back numbers are available.

Subscriptions, orders for back numbers, and changes of address should be sent to Pacific Journal of Mathematics, 103 Highland Boulevard, Berkeley, California, 94708.

PUBLISHED BY PACIFIC JOURNAL OF MATHEMATICS, A NON-PROFIT CORPORATION

Printed at Kokusai Bunken Insatsusha (International Academic Printing Co., Ltd.), 7-17, Fujimi 2-chome, Chiyoda-ku, Tokyo, Japan. 


\section{Pacific Journal of Mathematics}

\section{Vol. 37, No. $2 \quad$ February, 1971}

Charles Compton Alexander, Semi-developable spaces and quotient images of metric spaces .................................... 277

Ram Prakash Bambah and Alan C. Woods, On a problem of Danzer. . . . . . . . . 295

John A. Beekman and Ralph A. Kallman, Gaussian Markov expectations and related integral equations . ....................................

Frank Michael Cholewinski and Deborah Tepper Haimo, Inversion of the Hankel

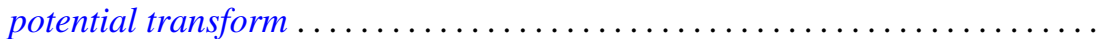

John H. E. Cohn, The diophantine equation

$$
Y(Y+1)(Y+2)(Y+3)=2 X(X+1)(X+2)(X+3) \ldots \ldots \ldots \ldots \ldots
$$

Philip C. Curtis, Jr. and Henrik Stetkaer, A factorization theorem for analytic

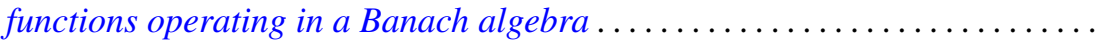

Doyle Otis Cutler and Paul F. Dubois, Generalized final rank for arbitrary limit ordinals

Keith A. Ekblaw, The functions of bounded index as a subspace of a space of entire functions

Dennis Michael Girard, The asymptotic behavior of norms of powers of

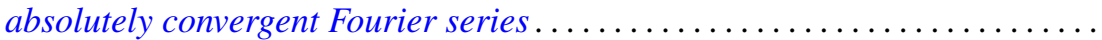

John Gregory, An approximation theory for elliptic quadratic forms on Hilbert spaces: Application to the eigenvalue problem for compact quadratic forms. 383

Paul C. Kainen, Universal coefficient theorems for generalized homology and stable cohomotopy.

Aldo Joram Lazar and James Ronald Retherford, Nuclear spaces, Schauder bases, and Choquet simplexes.

David Lowell Lovelady, Algebraic structure for a set of nonlinear integral operations

John McDonald, Compact convex sets with the equal support property . 429

Forrest Miller, Quasivector topologies

Marion Edward Moore and Arthur Steger, Some results on completability in commutative rings.

A. P. Morse, Taylor's theorem

Richard E. Phillips, Derek J. S. Robinson and James Edward Roseblade, Maximal subgroups and chief factors of certain generalized soluble groups.

Doron Ravdin, On extensions of homeomorphisms to homeomorphisms ...

John William Rosenthal, Relations not determining the structure of $\mathrm{L}$

Prem Lal Sharma, Proximity bases and subbases ........... .

Larry Smith, On ideals in $\Omega_{*}^{u}$. .

Warren R. Wogen, von Neumann algebras generated by operators similar to normal operators

R. Grant Woods, Co-absolutes of remainders of Stone-Čech 